\title{
QUEEN'S
UNIVERSITY
BELFAST
}

\section{A prospective study of circulating chemokines and angiogenesis markers and risk of multiple myeloma and its precursor}

Hofmann, J. N., Landgren, O., Landy, R., Kemp, T. J., Santo, L., McShane, C., Shearer, J. J., Lan, Q., Rothman, N., Pinto, L. A., Pfeiffer, R. M., Hildesheim, A., Katki, H. A., \& Purdue, M. P. (2019). A prospective study of circulating chemokines and angiogenesis markers and risk of multiple myeloma and its precursor. JNCI Cancer Spectrum. https://doi.org/10.1093/jncics/pkz104

Published in:

JNCI Cancer Spectrum

Document Version:

Peer reviewed version

Queen's University Belfast - Research Portal:

Link to publication record in Queen's University Belfast Research Portal

Publisher rights

Copyright 2019 OUP. This work is made available online in accordance with the publisher's policies. Please refer to any applicable terms of use of the publisher.

\section{General rights}

Copyright for the publications made accessible via the Queen's University Belfast Research Portal is retained by the author(s) and / or other copyright owners and it is a condition of accessing these publications that users recognise and abide by the legal requirements associated with these rights.

Take down policy

The Research Portal is Queen's institutional repository that provides access to Queen's research output. Every effort has been made to ensure that content in the Research Portal does not infringe any person's rights, or applicable UK laws. If you discover content in the Research Portal that you believe breaches copyright or violates any law, please contact openaccess@qub.ac.uk. 
A prospective study of circulating chemokines and angiogenesis markers and risk of multiple myeloma and its precursor

Jonathan N Hofmann ${ }^{1}$, Ola Landgren ${ }^{2}$, Rebecca Landy ${ }^{1}$, Troy J Kemp ${ }^{3}$, Loredana Santo ${ }^{1}$, Charlene M McShane ${ }^{4}$, Joseph J Shearer ${ }^{1}$, Qing Lan ${ }^{1}$, Nathaniel Rothman ${ }^{1}$, Ligia A Pinto ${ }^{3}$, Ruth M Pfeiffer ${ }^{1}$, Allan Hildesheim ${ }^{1}$, Hormuzd A Katki ${ }^{1}$, Mark P Purdue ${ }^{1}$

${ }^{1}$ Division of Cancer Epidemiology and Genetics, National Cancer Institute, Bethesda, MD

${ }^{2}$ Myeloma Service, Memorial Sloan-Kettering Cancer Center, New York, NY

${ }^{3}$ Frederick National Laboratory for Cancer Research, Leidos Biomedical Research, Inc., Frederick MD

${ }^{4}$ Cancer Epidemiology and Health Services Research Group, Centre for Public Health, Queen's University, Belfast, Northern Ireland, United Kingdom

\section{Correspondence to:}

Jonathan N Hofmann, PhD, MPH

Occupational and Environmental Epidemiology Branch

Division of Cancer Epidemiology and Genetics

National Cancer Institute

9609 Medical Center Drive

Room 6E604, MSC 9771

Bethesda, MD 20892

Phone: 240-276-7168

Fax: 240-276-7835

Email: hofmannjn@mail.nih.gov

Short title: Immunologic markers and multiple myeloma

Word count (abstract): 250

Word count (text): 2,964

Key words: multiple myeloma, monoclonal gammopathy of undetermined significance, chemokines, angiogenesis

Published by Oxford University Press 2019. This work is written by ( a) US Government employee(s) and is in the public domain in the US. 


\begin{abstract}
Background: Experimental and clinical studies have implicated certain chemokines and angiogenic cytokines in multiple myeloma (MM) pathogenesis. To investigate whether systemic concentrations of these markers are associated with future MM risk and progression from its precursor, monoclonal gammopathy of undetermined significance (MGUS), we conducted a prospective study within the Prostate, Lung, Colorectal and Ovarian Cancer Screening Trial.
\end{abstract} Methods: We measured concentrations of 45 immunologic and pro-angiogenic markers in sera from $241 \mathrm{MM}$ cases, 441 subjects with non-progressing MGUS, and 258 MGUS-free controls using Luminex-based multiplex assays and ELISA. Odds ratios (ORs) and 95\% confidence intervals (CIs) were estimated using multivariable logistic regression. We also evaluated absolute risk of progression using weighted Kaplan-Meier estimates.

Results: Pre-diagnostic levels of six markers were significantly elevated among MM cases compared with MGUS-free controls using a false discovery rate of 10\% (EGF, HGF, Ang-2, CXCL12, CCL8, and BMP-9). Of these, three angiogenesis markers were associated with future progression from MGUS to MM: EGF (fourth vs. first quartile: OR=3.01, 95\% CI 1.61 to 5.63; $\left.P_{\text {trend }}=0.00028\right), \operatorname{HGF}\left(2.59,1.33\right.$ to $\left.5.03 ; P_{\text {trend }}=0.015\right)$ and Ang-2 $(2.14,1.15$ to 3.98 ; $\left.P_{\text {trend }}=0.07\right)$. A composite angiogenesis biomarker score substantially stratified risk of MGUS progression to $\mathrm{MM}$ beyond established risk factors for progression, particularly during the first 5 years of follow-up (AUCs of 0.71 and 0.64 with and without the angiogenesis marker score, respectively).

Conclusions: Our prospective findings provide new insights into mechanisms involved in MM development and suggest that systemic angiogenesis markers could potentially improve risk stratification models for MGUS patients. 


\section{Introduction}

Multiple myeloma (MM) is a B cell malignancy characterized by the accumulation of malignant plasma cells in the bone marrow. Despite recent advancements in treatment, the prognosis for MM remains unfavorable, with an estimated 5-year relative survival rate of 52\% in the United States (1). MM is consistently preceded by a largely asymptomatic precursor condition called monoclonal gammopathy of undetermined significance (MGUS), which is typically characterized by detection of a monoclonal (M)-protein in serum and by measuring serum levels of kappa and lambda free light-chains (2-4). An abnormal serum free light-chain (sFLC) ratio in the absence of detectable M-protein is defined as light-chain (LC) MGUS, which can progress to LC MM (5). The likelihood of progression from MGUS to MM varies by certain characteristics of MGUS including the amount and type of M-protein and free light-chain levels/ratio, ranging from an estimated $0.3 \%$ progression per year for LC MGUS to approximately $1.5 \%$ per year for high-risk MGUS $(3,4)$. Improving the ability to identify MGUS patients at highest and lowest risk of progression could better inform clinical decision making regarding the frequency of follow-up testing and whether patients should be referred for more intensive procedures (e.g., bone marrow biopsy, skeletal imaging) (6).

Severe immune dysregulation is a strong risk factor for MM, with clearly elevated risks among solid organ transplant recipients taking immunosuppressive medications and HIV/AIDS patients (7-10). It is also suspected that subclinical variation in immune system function may influence risk in the general population. Mechanistic and clinical studies suggest that certain chemokines and pro-angiogenic growth factors play an important role in MM pathogenesis, and may hold promise as therapeutic targets (11-14). To our knowledge, however, no prospective 
studies have investigated the relationships between these markers and future MM risk and progression to MM among individuals with MGUS.

To address this research gap, we conducted a nested case-control study within the Prostate, Lung, Colorectal and Ovarian (PLCO) Cancer Screening Trial measuring levels of chemokines, pro-angiogenic cytokines and other immunologic markers in banked sera from three study groups: subjects newly diagnosed with MM during follow-up, subjects with MGUS who have not progressed to MM, and MGUS-free subjects. Our aims were to investigate marker associations with (1) future MM risk and (2) future progression from MGUS to MM. For selected markers, we additionally evaluated the potential improvement in prediction of absolute risk of progression from MGUS to MM beyond factors in the current Mayo risk stratification model (2-4).

\section{Methods}

Study population

The design of the PLCO Cancer Screening Trial has been reported (15). The current investigation leveraged data from a larger screening study of MGUS in PLCO (16), described in the Supplemental Methods. A schematic diagram outlining the design of the current nested casecontrol study is shown in Supplemental Figure 1. Briefly, MGUS status was assessed in banked sera from MM cases diagnosed during follow-up and a large sample $(\mathrm{N}=5,916)$ of participants who did not develop MM or other hematologic malignancies, selected through stratified random sampling based on age, sex, and race. Our study included 241 newly diagnosed MM cases with available banked serum samples (185 of which were 'progressors' with MGUS characterized at the time of selection), 441 stable non-IgM or LC MGUS that had not progressed to MM through 
up to 19 years of follow-up, and 258 MGUS-free controls. Cases of IgM MGUS were not included in this investigation, as this isotype generally progresses to a different malignancy, Waldenström macroglobulinemia $(3,4)$. MGUS-free controls were matched to MM cases on age at blood collection ( \pm 2 years), sex, race (non-Hispanic white, black, other), date and study year of phlebotomy, and number of freeze-thaw cycles. For participants with detected MGUS, we selected the earliest available banked serum sample at which MGUS was identified for use in this study. For MM cases without pre-diagnostic information on MGUS (N=56), we selected the earliest available serum sample. The PLCO Cancer Screening Trial was approved by Institutional Review Boards at the National Cancer Institute and the 10 study centers, and all participants provided written informed consent.

\section{Immune marker measurements}

Serum levels of 46 chemokines and angiogenesis markers were measured using three Millipore multiplex bead-based assay panels, and two soluble CD activation markers (sCD27, sCD30) were measured using standard ELISA methods. Samples were tested in duplicate for the angiogenesis panel and ELISA assays, and in single wells for the chemokine panels. We excluded three markers with $>90 \%$ of test samples below the lower limit of quantification (LLOQ; CCL21, FGF-1, and IL-3), leaving 45 markers for analysis. For these remaining markers, measurements below the LLOQ were assigned half of the LLOQ value. Levels were detectable in $>90 \%$ of samples for most markers $(\mathrm{N}=29,64 \%)$, and assays demonstrated good reproducibility; the median coefficient of variation was $8.5 \%$, and all intraclass correlation coefficients were $\geq 0.8$ (Supplemental Table 1). 


\section{Statistical analysis}

We used polytomous logistic regression models to estimate odds ratios (OR) and 95\% confidence intervals $(\mathrm{CI})$ relating concentrations of immunologic markers and risks of MGUS and MM relative to MGUS-free controls; all models were adjusted for age, sex, race, study and calendar year of phlebotomy, and study center. Adjustment for body mass index (BMI) did not materially impact our results, and as such BMI was not included in our final statistical models. Markers were analyzed as continuous variables after $\log _{2}$ transformation such that risk estimates correspond to a doubling in marker levels. Associations with MM were considered statistically significant if the two-sided $P$-value was $<0.05$ and the Benjamini-Hochberg false discovery rate (FDR) was $<10 \%$; FDR calculations were based on a total of 45 markers evaluated in the primary analyses.(17) Further categorical analyses of the top markers were performed with category cut points based on the quartiles among controls. We also conducted analyses stratified by sex, MGUS type (LC vs. heavy-chain non-IgM MGUS), time from blood collection to MM diagnosis based on median follow-up ( $<8$ years, $\geq 8$ years), and sensitivity analyses restricted to non-Hispanic whites.

For markers associated with MM risk, we also evaluated associations with progression among those with MGUS who later developed MM versus those with stable MGUS that did not progress to MM during follow-up. We computed ORs and 95\% CIs using unconditional logistic regression models adjusted for the same factors as above, and further adjusted for established risk factors for progression modeled as a 3-category variable based on the current Mayo risk stratification model: LC MGUS, low risk non-IgM MGUS (M-protein concentration not elevated, normal sFLC ratio), and intermediate/high risk non-IgM MGUS (M-protein concentration $\geq 15 \mathrm{~g} / \mathrm{L}$ and/or sFLC ratio $<0.26$ or $>1.65)$.(2-4) For these analyses, quartile cut 
points were assigned based on the distribution among the referent group of those with stable MGUS that did not progress to MM. We also conducted analyses stratified by sex and time from detection of MGUS/marker measurements to MM diagnosis ( $<5$ years, $\geq 5$ years).

We estimated the cumulative risk of progression from MGUS to MM (complement of Kaplan-Meier survival estimates) by weighting the nested case-control sample to represent the PLCO screening arm participants using the R package NestedCohort. The weights for membership in the nested case-control study depended on 48 cells crossing MGUS/MM status, age (55-59, 60-64, 65-69, and $\geq 70$ years), sex, and race/ethnicity. A composite angiogenesis marker score was developed using a logistic regression model of MGUS progression in relation to $\log _{2}$-transformed concentrations of EGF, HGF, and Ang-2. This score is the sum of the $\log _{2}$ marker concentration multiplied by its regression coefficient. Scores were dichotomized into the top tertile versus the two lower tertiles to compare absolute risks of progression. We assessed the improvement in predictions of absolute risk beyond the current Mayo risk stratification model by including the composite angiogenesis score versus only the 3-category MGUS variable defined above using Mean Risk Stratification (MRS), which estimates the average change in MM risk revealed by the angiogenesis score (18). MRS interprets the area-under-the-curve (AUC) in light of disease prevalence; a high AUC with low MM prevalence might have less risk stratification than a low AUC with high MM prevalence.

Statistical analyses were performed using Stata version 15.0 (College Station, TX) and R version 3.5 .

\section{Results}

Demographic characteristics of study participants are shown in Table 1. A greater proportion of the MGUS cases were black relative to MM cases and controls, as expected based 
on the sampling strategy for MGUS screening which over-sampled black participants, as described. Other demographic characteristics were generally similar for MM, MGUS, and controls. Among MM cases, the median time from sample collection to diagnosis was 8.3 years (range $<1-19$ years).

Figure 1 summarizes the $P$-values for tests of association with MM risk relative to MGUS-free controls for all 45 markers modeled as continuous variables. We identified six markers - four angiogenic cytokines/growth factors (EGF, HGF, Ang-2, BMP-9) and two chemokines (CXCL12, CCL8) - that were statistically significantly associated with MM risk using a FDR of $10 \%$. These markers were uncorrelated or only modestly correlated with one another or with characteristics of MGUS (M-protein concentration, sFLC levels and ratio) (Supplemental Table 2). As shown in Table 2, all six markers were positively associated with MM risk. These associations remained for cases diagnosed $\geq 8$ years after blood collection for all markers except CCL8 (Supplemental Table 3) and did not materially differ by sex (results not shown).

In analyses comparing stable MGUS cases and MGUS-free controls (Table 2), we observed strong associations with the chemokines CXCL12 and CCL8, although tests for trend were also statistically significant for HGF and Ang-2. While results for LC MGUS and non-IgM MGUS were not significantly different, associations with CXCL12, HGF, and Ang-2 were more apparent among those with non-IgM MGUS and CCL8 was more strongly associated with LC MGUS (Supplemental Table 4). Comparisons of marker levels among stable MGUS and MM cases, respectively, versus MGUS-free controls are shown in Supplemental Figure 2.

When we compared MM 'progressors' and stable MGUS, we observed an increased risk of future progression to MM among those with high levels of the angiogenic cytokines EGF 
(fourth quartile vs. first: $\mathrm{OR}=3.01,95 \% \mathrm{CI} 1.61$ to $\left.5.63, P_{\text {trend }}=2.8 \times 10^{-4}\right)$, $\operatorname{HGF}(2.59,1.33$ to 5.03, $\left.P_{\text {trend }}=0.015\right)$, and Ang-2 $\left(2.14,1.15\right.$ to $3.98, P_{\text {trend }}=0.07$; Table 3$)$. Associations with HGF and Ang-2 were particularly strong for MM cases that progressed within 5 years of blood draw relative to those diagnosed later $\left(P_{\text {heterogeneity }} \leq 0.0036\right)$, whereas associations with EGF did not appear to vary by follow-up time. Risk estimates were generally similar in magnitude for sensitivity analyses restricted to non-Hispanic whites and stratified by sex (data not shown).

Figure 2 shows the absolute risk of progression from MGUS to MM for three established MGUS risk groups (LC MGUS, low risk non-IgM MGUS, and intermediate/high risk non-IgM MGUS) and a composite angiogenesis marker score based on EGF, HGF, and Ang-2. In each risk group, risk of 15-year progression was increased among those with an angiogenesis score in the top tertile vs. those in lower tertiles (LC MGUS: $4.2 \%$ vs $1.0 \%, P=0.016$; low-risk non-IgM MGUS: $8.2 \%$ vs. $3.5 \%, P=0.056$; intermediate/high-risk non-IgM MGUS: $18.3 \%$ vs. $8.9 \%$, $P=0.015$ ). Differences in progression risk were particularly notable during the first five years of follow-up (LC MGUS: $2.6 \%$ vs $0.2 \%, P=0.012$; low-risk non-IgM MGUS: $2.3 \%$ vs. $0.6 \%$, $P=0.10$; intermediate/high-risk non-IgM MGUS: $8.3 \%$ vs. $1.4 \%, P=0.0006)$.

In the first 5 years of follow-up, the overall AUC for progression from MGUS to MM increased from 0.64 (using risk strata in the current Mayo model) to 0.71 after incorporating the composite angiogenesis marker score. Within individual MGUS risk strata, the AUCs for the angiogenesis score at 5 years of follow-up ranged from 0.65 to 0.78 , with $52 \%-83 \%$ of MM diagnosed during this time period occurring among those with high angiogenesis score (Table 4). The ability of the angiogenesis score to predict progression between 5-10 years of follow-up was attenuated; compared to the first 5 years, the AUCs declined from $0.65-0.78$ to $0.54-0.58$, the 
fraction of $\mathrm{MM}$ with a high angiogenesis score declined from $52 \%-83 \%$ to $36 \%-43 \%$, and the MRS declined from $0.11 \%-0.61 \%$ to $0.07 \%-0.18 \%$ (Table 4 ).

The composite angiogenesis marker score performed better for risk prediction than any of the three angiogenesis markers modeled individually (Supplementary Figure 3) and yielded similar findings using alternate cut points (e.g., top quartile vs. lower quartiles; Supplementary Figure 4).

\section{Discussion}

This study is, to our knowledge, the largest prospective investigation of circulating chemokines and pro-angiogenic cytokines in relation to risk of MM and the first to evaluate whether these markers are associated with future progression from MGUS to MM. In casecontrol analyses we observed strong positive associations with future MM risk for four proangiogenic cytokines/growth factors (EGF, HGF, Ang-2, and BMP-9) and two chemokines (CXCL12 and CCL8) after accounting for multiple comparisons. Of the six markers associated with future MM risk, we observed particularly strong associations with progression from MGUS to $\mathrm{MM}$ in relation to three pro-angiogenic cytokines (EGF, HGF, and Ang-2). Notably, a composite angiogenesis marker score based on levels of these three markers substantially improved our ability to predict absolute risk of progression from MGUS to MM beyond criteria in the current Mayo risk stratification model,(2-4) particularly during the first five years of follow-up. Two recent prospective studies with fewer MM cases (N=76 and 65, respectively) identified several immune markers that were inversely associated with MM risk $(19,20)$; however, these studies did not evaluate most of the markers that we found to be positively associated with MM risk and MGUS progression to MM, and other overlapping markers (e.g., FGF-2, VEGF, TGF- $\alpha$ ) did not reach statistical significance in our analyses. 
Increased bone marrow angiogenesis plays a well-established role in MGUS progression to MM (12-14), and the transition to a vascular phase of tumor growth has been defined as an 'angiogenic switch' in the equilibrium between positive and inhibitory regulators of angiogenic activity that is characteristic of progression to active MM (21-24). Elevated angiogenesis markers - including those associated with future progression from MGUS to MM in this study have also been linked to poorer prognosis among MM patients $(13,22,25,26)$, and, more recently, with response to treatment (27). The EGF family of proteins plays a critical role in bone biology and have been studied as myeloma growth factors $(28,29)$. EGF enhances proliferation and migration in bone marrow stromal cells and directly targets the pro-inflammatory cytokine IL-6 (30), which has been shown to influence MM pathogenesis (31). Increased expression of HGF and Ang-2 has been correlated with more advanced disease stage and poorer prognosis $(13,25,26,32,33)$; elevated levels of these markers may reflect hypoxia and enhanced angiogenic activity in the bone marrow microenvironment, which contributes to disease progression and tumor dissemination (12). Although increased expression of VEGF in the bone marrow is thought to play an important role in MM pathogenesis (34), we did not observe associations with circulating levels of VEGF for MM risk or MGUS progression to MM; ex vivo platelet degranulation can affect VEGF measurements in serum (35), which may have limited our ability to detect associations with this marker.

Our findings suggest that a composite angiogenesis marker score may improve risk stratification for progression from MGUS to MM based on the existing Mayo model (2-4). Interestingly, we observed statistically significant differences in progression to MM between subjects with high versus low levels of the dichotomized biomarker score among those with intermediate/high-risk non-IgM MGUS as well as for those with low-risk LC MGUS, suggesting 
potential improvements in stratification at both ends of the current risk spectrum. Across MGUS risk strata, the ability of the angiogenesis score to predict progression to MM was most apparent during the first 5 years of follow-up, and was attenuated $\geq 5$ years after assessment of MGUS and the angiogenesis markers. Assessment of longitudinal changes in these angiogenesis markers could potentially improve our ability to discriminate the risk of progression over a longer period of follow-up. Furthermore, we note that if our findings are confirmed in a validation set, these markers could potentially be used to improve existing risk stratification models for progression from MGUS to MM, and as such may have clinical utility for better informing decisions about the frequency and type of follow-up testing over time.

Whereas the associations with progression from MGUS to MM were most apparent for these angiogenesis markers, we found that the chemokines CXCL12 and CCL8 were most strongly associated with MGUS, suggesting that these markers may reflect mechanisms involved at the early stages of MM development. CXCL12 is secreted by mesenchymal stromal cells and other cell types in the bone marrow, and it binds to the CXCR4 receptor, which is expressed on myeloma cells (12). Findings from experimental studies suggest that this chemokine supports the migration, adhesion, and homing of myeloma cells to the bone marrow $(12,36)$. CCL8 is also produced by bone marrow stromal cells and it targets the CCR2 receptor, another chemokine receptor expressed by myeloma cells (37). Experimental data for CCL8 are less robust but suggest that this chemokine may also be involved in bone marrow homing of myeloma cells $(11,37)$.

A unique feature of this study is the availability of information on MGUS status for the majority of MM cases and the inclusion of stable MGUS that did not progress to MM during follow-up. This allowed us to directly investigate markers associated with progression from 
MGUS to MM and assess their potential utility for improving existing risk stratification models. While we used a conservative approach to control for multiple comparisons, and there is strong biologic plausibility for the observed associations, confirmation of these findings in other prospective studies is warranted. In this investigation we utilized data on MGUS and measured chemokines and angiogenesis markers in samples at a single time point prior to $\mathrm{MM}$ diagnosis/selection; future longitudinal investigations using measurements in serial samples collected over time may provide greater insights into whether marker trajectories predict MGUS progression to MM. Limitations of the current study include the small number of MM cases for certain subgroup analyses (e.g., progression from LC MGUS to LC MM, and in analyses stratified by follow-up time) and the absence of specimens to characterize clinically distinct MM tumor subtypes (e.g., hyperdiploid vs. non-hyperdiploid, IGH translocations) or identify cases of smoldering MM. Efforts to validate our findings in larger datasets with more detailed information on clinical characteristics of MM would be informative.

In summary, this prospective investigation of MM, its precursor MGUS, and MGUS-free controls identified six circulating immunologic markers associated with future MM risk, offering new insights into mechanisms involved in MM development. In particular, our findings provide novel evidence that systemic markers of angiogenesis are predictive of increased future risk of progression from MGUS to clinically manifest MM. Additional prospective studies are needed to replicate our findings; if confirmed, these markers could substantially improve our ability to identify MGUS patients at high and low risk of progression and may help inform decision making regarding clinical management of MGUS.

\section{Acknowledgements}


This work was supported by the Intramural Research Program of the U.S. National Cancer Institute (NCI), National Institutes of Health (NIH). The NCI funded the PLCO Cancer Screening Trial, which was also supported by contracts from the Division of Cancer Prevention of the NCI and by the Intramural Research Program of the Division of Cancer Epidemiology and Genetics, NCI, NIH. The authors thank the PLCO Cancer Screening Trial screening center investigators; Michael Furr, Craig Williams, Shannon Merkle, Michael Curry, and other staff at Information Management Services, Inc.; and, most importantly, the study participants for their contributions that made this study possible.

\section{Conflicts of Interest}

OL has received research funding from: National Institutes of Health (NIH), U.S. Food and Drug Administration (FDA), Multiple Myeloma Research Foundation (MMRF), International Myeloma Foundation (IMF), Leukemia and Lymphoma Society (LLS), Perelman Family Foundation, Rising Tides Foundation, Amgen, Celgene, Janssen, Takeda, Glenmark, Seattle Genetics, Karyopharm; Honoraria/ad boards: Adaptive, Amgen, Binding Site, BMS, Celgene, Cellectis, Glenmark, Janssen, Juno, Pfizer; and serves on Independent Data Monitoring Committees (IDMCs) for clinical trials lead by Takeda, Novartis, Janssen. The other authors declare no competing financial interests. 


\section{References}

1. ACS. Cancer Facts \& Figures 2019, American Cancer Society: Atlanta, GA. Atlanta, GA: American Cancer Society;2019.

2. Kyle RA, Durie BG, Rajkumar SV, et al. Monoclonal gammopathy of undetermined significance (MGUS) and smoldering (asymptomatic) multiple myeloma: IMWG consensus perspectives risk factors for progression and guidelines for monitoring and management. Leukemia. 2010;24(6):1121-1127.

3. Rajkumar SV, Dimopoulos MA, Palumbo A, et al. International Myeloma Working Group updated criteria for the diagnosis of multiple myeloma. The Lancet Oncology. 2014;15(12):e538-e548.

4. Kyle RA, Larson DR, Therneau TM, et al. Long-Term Follow-up of Monoclonal Gammopathy of Undetermined Significance. N Engl J Med. 2018;378(3):241-249.

5. Dispenzieri A, Katzmann JA, Kyle RA, et al. Prevalence and risk of progression of lightchain monoclonal gammopathy of undetermined significance: a retrospective populationbased cohort study. Lancet. 2010;375(9727):1721-1728.

6. Landgren O, Waxman AJ. Multiple myeloma precursor disease. JAMA. 2010;304(21):2397-2404.

7. Engels EA, Pfeiffer RM, Fraumeni JF, et al. Spectrum of cancer risk among US solid organ transplant recipients. JAMA. 2011;306(17):1891-1901.

8. Goedert JJ, Cote TR, Virgo P, et al. Spectrum of AIDS-associated malignant disorders. Lancet. 1998;351(9119):1833-1839. 
9. Grulich AE, van Leeuwen MT, Falster MO, Vajdic CM. Incidence of cancers in people with HIV/AIDS compared with immunosuppressed transplant recipients: a meta-analysis. Lancet. 2007;370(9581):59-67.

10. Quinlan SC, Morton LM, Pfeiffer RM, et al. Increased risk for lymphoid and myeloid neoplasms in elderly solid-organ transplant recipients. Cancer Epidemiol Biomarkers Prev. 2010;19(5):1229-1237.

11. Aggarwal R, Ghobrial IM, Roodman GD. Chemokines in multiple myeloma. Exp Hematol. 2006;34(10):1289-1295.

12. Ghobrial IM, Detappe A, Anderson KC, Steensma DP. The bone-marrow niche in MDS and MGUS: implications for AML and MM. Nat Rev Clin Oncol. 2018;15(4):219-233.

13. Jakob C, Sterz J, Zavrski I, et al. Angiogenesis in multiple myeloma. Eur J Cancer. 2006;42(11):1581-1590.

14. Kumar S, Witzig TE, Timm M, et al. Bone marrow angiogenic ability and expression of angiogenic cytokines in myeloma: evidence favoring loss of marrow angiogenesis inhibitory activity with disease progression. Blood. 2004;104(4):1159-1165.

15. Hayes RB, Reding D, Kopp W, et al. Etiologic and early marker studies in the prostate, lung, colorectal and ovarian (PLCO) cancer screening trial. Control Clin Trials. 2000;21(6 Suppl):349S-355S.

16. Landgren O, Hofmann JN, McShane CM, et al. Association of immune marker changes with progression of monoclonal gammopathy of undetermined significance to multiple myeloma. JAMA Oncology. 2019 Jul 18 (Epub ahead of print).

17. Benjamini Y, Hochberg Y. Controlling the False Discovery Rate: a Practical and Powerful Approach to Multiple Testing. J R Statist Soc B. 1995;57(1):289-300. 
18. Katki HA, Schiffman M. A novel metric that quantifies risk stratification for evaluating diagnostic tests: The example of evaluating cervical-cancer screening tests across populations. Prev Med. 2018;110:100-105.

19. Vermeulen R, Saberi Hosnijeh F, Bodinier B, et al. Pre-diagnostic blood immune markers, incidence and progression of B-cell lymphoma and multiple myeloma: Univariate and functionally informed multivariate analyses. Int J Cancer. 2018;143(6):1335-1347.

20. Spath F, Wibom C, Krop EJM, et al. Immune marker changes and risk of multiple myeloma: a nested case-control study using repeated prediagnostic blood samples. Haematologica. 2019 Apr 04 (Epub ahead of print).

21. Vacca A, Ribatti D, Roncali L, et al. Bone marrow angiogenesis and progression in multiple myeloma. Br J Haematol. 1994;87(3):503-508.

22. Vacca A, Ribatti D. Bone marrow angiogenesis in multiple myeloma. Leukemia. 2006;20(2):193-199.

23. Ribatti D, Nico B, Crivellato E, Roccaro AM, Vacca A. The history of the angiogenic switch concept. Leukemia. 2007;21(1):44-52.

24. Giuliani N, Storti P, Bolzoni M, Palma BD, Bonomini S. Angiogenesis and multiple myeloma. Cancer Microenviron. 2011;4(3):325-337.

25. Pappa CA, Tsirakis G, Samiotakis P, et al. Serum levels of angiopoietin-2 are associated with the growth of multiple myeloma. Cancer investigation. 2013;31(6):385-389.

26. Seidel C, Borset M, Turesson I, Abildgaard N, Sundan A, Waage A. Elevated serum concentrations of hepatocyte growth factor in patients with multiple myeloma. The Nordic Myeloma Study Group. Blood. 1998;91(3):806-812. 
27. Saltarella I, Morabito F, Giuliani N, et al. Prognostic or predictive value of circulating cytokines and angiogenic factors for initial treatment of multiple myeloma in the GIMEMA MM0305 randomized controlled trial. J Hematol Oncol. 2019;12(1):4.

28. Mahtouk K, Moreaux J, Hose D, et al. Growth factors in multiple myeloma: a comprehensive analysis of their expression in tumor cells and bone marrow environment using Affymetrix microarrays. BMC Cancer. 2010;10:198.

29. Cao Y, Luetkens T, Kobold S, et al. The cytokine/chemokine pattern in the bone marrow environment of multiple myeloma patients. Exp Hematol. 2010;38(10):860-867.

30. Muller-Deubert S, Seefried L, Krug M, Jakob F, Ebert R. Epidermal growth factor as a mechanosensitizer in human bone marrow stromal cells. Stem Cell Res. 2017;24:69-76.

31. Klein B, Tarte K, Jourdan M, et al. Survival and proliferation factors of normal and malignant plasma cells. Int J Hematol. 2003;78(2):106-113.

32. Bhutani M, Turkbey B, Tan E, et al. Bone marrow angiogenesis in myeloma and its precursor disease: a prospective clinical trial. Leukemia. 2014;28(2):413-416.

33. Belloni D, Marcatti M, Ponzoni M, et al. Angiopoietin-2 in Bone Marrow milieu promotes Multiple Myeloma-associated angiogenesis. Exp Cell Res. 2015;330(1):1-12.

34. Rajkumar SV, Mesa RA, Fonseca R, et al. Bone marrow angiogenesis in 400 patients with monoclonal gammopathy of undetermined significance, multiple myeloma, and primary amyloidosis. Clin Cancer Res. 2002;8(7):2210-2216.

35. Engels EA, Jennings L, Kemp TJ, et al. Circulating TGF-beta1 and VEGF and risk of cancer among liver transplant recipients. Cancer Med. 2015;4(8):1252-1257.

36. Bouyssou JM, Ghobrial IM, Roccaro AM. Targeting SDF-1 in multiple myeloma tumor microenvironment. Cancer Lett. 2016;380(1):315-318. 
37. Vande Broek I, Asosingh K, Vanderkerken K, Straetmans N, Van Camp B, Van Riet I. Chemokine receptor CCR2 is expressed by human multiple myeloma cells and mediates migration to bone marrow stromal cell-produced monocyte chemotactic proteins MCP-1, -2 and -3. British journal of cancer. 2003;88(6):855-862.

38. Cantor SB, Kattan MW. Determining the area under the ROC curve for a binary diagnostic test. Med Decis Making. 2000;20(4):468-470. 
Table 1. Demographic characteristics of MGUS and MM cases and MGUS-free controls*

\begin{tabular}{llll}
\hline Characteristic & Controls & MGUS & Multiple myeloma \\
\hline $\begin{array}{l}\text { Overall } \\
\text { Age at blood draw, } \\
\text { median (IQR) }\end{array}$ & $258(59-69)$ & 441 & 241 \\
$\begin{array}{l}\text { Sex } \\
\text { Female }\end{array}$ & & $68(62-72)$ & $64(59-68)$ \\
$\quad$ Male & $89(35 \%)$ & $133(30 \%)$ & \\
Race & $169(66 \%)$ & $308(70 \%)$ & $84(35 \%)$ \\
Non-Hispanic white & $235(91 \%)$ & & $157(65 \%)$ \\
Black & $12(5 \%)$ & $271(61 \%)$ & $219(91 \%)$ \\
Other & $11(4 \%)$ & $143(32 \%)$ & $12(5 \%)$ \\
MGUS subtype & & $27(6 \%)$ & $10(4 \%)$ \\
IgG & & $205(46 \%)$ & $107(58 \%)$ \\
IgA & & $42(10 \%)$ & $45(24 \%)$ \\
Biclonal & $17(4 \%)$ & $5(3 \%)$ \\
Light chain & & $177(40 \%)$ & $28(15 \%)$ \\
\hline
\end{tabular}

* Reported as frequency (\%) unless otherwise noted. 
Table 2. Odds ratios (95\% confidence intervals) for selected markers and risk of MGUS and MM in relation to MGUS-free controls*

\begin{tabular}{|c|c|c|c|c|c|}
\hline \multirow[b]{2}{*}{ Marker (pg/mL) } & \multirow[b]{2}{*}{$\mathrm{N}_{\text {controls }}$} & \multicolumn{2}{|r|}{ MGUS } & \multicolumn{2}{|r|}{ Multiple myeloma } \\
\hline & & $\mathrm{N}$ & OR $(95 \% \mathrm{CI})$ & $\mathrm{N}$ & OR $(95 \% \mathrm{CI})$ \\
\hline \multicolumn{6}{|l|}{ EGF } \\
\hline$<41.9$ & 65 & 110 & 1.00 (ref) & 41 & 1.00 (ref) \\
\hline $41.9-72.7$ & 65 & 79 & $0.83(0.50$ to 1.36$)$ & 30 & $0.99(0.53$ to 1.84$)$ \\
\hline $72.8-135.8$ & 64 & 121 & $1.17(0.72$ to 1.90$)$ & 52 & $1.73(0.97$ to 3.10$)$ \\
\hline$>135.8$ & 64 & 131 & $1.01(0.62$ to 1.64$)$ & 118 & $3.12(1.80$ to 5.42$)$ \\
\hline $\mathrm{P}_{\text {trend }}$ & & & 0.79 & & $2.9 \times 10^{-6}$ \\
\hline Continuous $^{\dagger}$ & 258 & 441 & $1.06(0.94$ to 1.19$)$ & 241 & $1.52(1.31$ to 1.76$)$ \\
\hline \multicolumn{6}{|l|}{ HGF } \\
\hline$<456.5$ & 66 & 118 & 1.00 (ref) & 39 & 1.00 (ref) \\
\hline $456.5-579.0$ & 64 & 78 & $0.69(0.41$ to 1.15$)$ & 39 & $1.13(0.62$ to 2.07$)$ \\
\hline $579.1-750.3$ & 64 & 99 & $0.90(0.55$ to 1.48$)$ & 69 & $2.26(1.28$ to 4.00$)$ \\
\hline$>750.3$ & 64 & 146 & $1.53(0.95$ to 2.44$)$ & 94 & $2.94(1.69$ to 5.11$)$ \\
\hline $\mathrm{P}_{\text {trend }}$ & & & 0.018 & & $2.5 \times 10^{-5}$ \\
\hline Continuous $^{\dagger}$ & 258 & 441 & 1.30 (1.02 to 1.67$)$ & 241 & $1.89(1.42$ to 2.51$)$ \\
\hline \multicolumn{6}{|l|}{ Ang-2 } \\
\hline$<1508.7$ & 66 & 84 & 1.00 (ref) & 33 & 1.00 (ref) \\
\hline $1508.7-2193.7$ & 64 & 92 & $1.00(0.60$ to 1.65$)$ & 56 & $1.70(0.94$ to 3.08$)$ \\
\hline 2193.8-3103.9 & 64 & 110 & $1.16(0.70$ to 1.91$)$ & 55 & $1.71(0.95$ to 3.08$)$ \\
\hline$>3103.9$ & 64 & 155 & $1.54(0.95$ to 2.49$)$ & 97 & $3.00(1.71$ to 5.26$)$ \\
\hline $\mathrm{P}_{\text {trend }}$ & & & 0.04 & & $1.8 \times 10^{-4}$ \\
\hline Continuous $^{\dagger}$ & 258 & 441 & $1.20(0.97$ to 1.47$)$ & 241 & $1.57(1.24$ to 1.98$)$ \\
\hline \multicolumn{6}{|l|}{ CXCL12 } \\
\hline$<1117.0$ & 65 & 74 & 1.00 (ref) & 39 & 1.00 (ref) \\
\hline $1117.0-1451.3$ & 65 & 91 & $1.25(0.75$ to 2.11$)$ & 50 & $1.27(0.71$ to 2.27$)$ \\
\hline $1451.4-1780.5$ & 64 & 114 & 1.84 (1.10 to 3.07$)$ & 54 & $1.56(0.87$ to 2.77$)$ \\
\hline$>1780.5$ & 64 & 162 & $2.36(1.44$ to 3.88$)$ & 98 & $2.62(1.52$ to 4.52$)$ \\
\hline $\mathrm{P}_{\text {trend }}$ & & & $2.4 \times 10^{-4}$ & & $2.6 \times 10^{-4}$ \\
\hline Continuous $^{\dagger}$ & 258 & 441 & $1.45(1.15$ to 1.84$)$ & 241 & $1.71(1.27$ to 2.30$)$ \\
\hline \multicolumn{6}{|l|}{ CCL8 } \\
\hline$<23.1$ & 64 & 67 & 1.00 (ref) & 38 & 1.00 (ref) \\
\hline $23.1-32.6$ & 66 & 114 & $1.79(1.07$ to 3.00$)$ & 64 & $1.74(0.98$ to 3.08$)$ \\
\hline $32.7-43.4$ & 64 & 107 & $1.96(1.17$ to 3.30$)$ & 67 & $1.86(1.05$ to 3.30$)$ \\
\hline$>43.4$ & 64 & 153 & 2.11 (1.26 to 3.52$)$ & 72 & $2.08(1.17$ to 3.70$)$ \\
\hline$P_{\text {trend }}$ & & & 0.011 & & 0.021 \\
\hline Continuous $^{\dagger}$ & 258 & 441 & $1.51(1.18$ to 1.93$)$ & 241 & 1.55 (1.17 to 2.04$)$ \\
\hline \multicolumn{6}{|l|}{ BMP-9 } \\
\hline$<101.3$ & 65 & 131 & 1.00 (ref) & 40 & 1.00 (ref) \\
\hline $101.3-146.9$ & 65 & 101 & $0.70(0.43$ to 1.14$)$ & 40 & $0.92(0.51$ to 1.67$)$ \\
\hline $147.0-218.8$ & 64 & 104 & $0.75(0.46$ to 1.21$)$ & 63 & $1.66(0.95$ to 2.90$)$ \\
\hline$>218.8$ & 64 & 105 & $0.93(0.57$ to 1.51$)$ & 98 & $2.31(1.34$ to 3.99$)$ \\
\hline $\mathrm{P}_{\text {trend }}$ & & & 0.94 & & $4.1 \times 10^{-4}$ \\
\hline Continuous $^{\dagger}$ & 258 & 441 & $0.93(0.82$ to 1.06$)$ & 241 & $1.29(1.09$ to 1.52$)$ \\
\hline
\end{tabular}

* All analyses were adjusted for age, sex, race, study year and calendar year of phlebotomy, and study center. Tests for trend were performed by assigning the value of the within-category median (among controls) and modeling as a continuous variable.

$\dagger$ Estimated risk corresponding to an approximate doubling in marker levels (1-unit increase in $\log _{2}$-transformed levels). 
Table 3. Odds ratios (95\% confidence intervals) for selected markers and risk of progression from MGUS to MM*

\begin{tabular}{|c|c|c|c|c|c|c|c|c|}
\hline \multirow{2}{*}{$\begin{array}{l}\text { Marker } \\
(\mathrm{pg} / \mathrm{mL})\end{array}$} & \multirow[b]{2}{*}{$\mathrm{N}_{\text {MGUS }}$} & \multicolumn{6}{|c|}{$<5$ years to $\mathrm{MM}$} & \multirow[b]{2}{*}{$P_{\text {het }}$} \\
\hline & & $\mathrm{N}_{\mathrm{MM}}$ & OR $(95 \% \mathrm{CI})$ & $\mathrm{N}_{\mathrm{MM}}$ & OR $(95 \% \mathrm{CI})$ & $\mathrm{N}_{\mathrm{MM}}$ & $\mathrm{OR}(95 \% \mathrm{CI})$ & \\
\hline \multicolumn{9}{|l|}{$\mathrm{EGF}$} \\
\hline$<42.5$ & 111 & 36 & 1.00 (ref) & 10 & 1.00 (ref) & 26 & 1.00 (ref) & \\
\hline $42.5-85.5$ & 110 & 37 & $1.36(0.71$ to 2.64$)$ & 7 & $0.94(0.31$ to 2.79$)$ & 30 & $1.54(0.76$ to 3.15$)$ & \\
\hline $85.6-149.5$ & 111 & 35 & $1.20(0.62$ to 2.31$)$ & 9 & $1.10(0.39$ to 3.12$)$ & 26 & $1.23(0.59$ to 2.54$)$ & \\
\hline$>149.5$ & 109 & 77 & $3.01(1.61$ to 5.63$)$ & 32 & $3.62(1.47$ to 8.91$)$ & 45 & $2.71(1.35$ to 5.44$)$ & \\
\hline$P_{\text {trend }}$ & & & $2.8 \times 10^{-4}$ & & 0.0006 & & 0.0056 & \\
\hline Continuous $^{\dagger}$ & 441 & 185 & $1.31(1.12$ to 1.54$)$ & 58 & $1.47(1.15$ to 1.87$)$ & 127 & $1.25(1.05$ to 1.49$)$ & 0.22 \\
\hline \multicolumn{9}{|l|}{ HGF } \\
\hline$<443.0$ & 111 & 26 & 1.00 (ref) & 3 & $1.00(\mathrm{ref})$ & 23 & 1.00 (ref) & \\
\hline $443.0-621.3$ & 110 & 47 & $2.24(1.12$ to 4.46$)$ & 9 & $3.38(0.82$ to 14.0$)$ & 38 & $2.04(0.99$ to 4.21$)$ & \\
\hline $621.4-812.7$ & 110 & 54 & $2.69(1.38$ to 5.24$)$ & 14 & $6.16(1.59$ to 23.8$)$ & 40 & $2.16(1.07$ to 4.39$)$ & \\
\hline$>812.7$ & 110 & 58 & $2.59(1.33$ to 5.03$)$ & 32 & $12.02(3.30$ to 43.9$)$ & 26 & $1.33(0.63$ to 2.80$)$ & \\
\hline $\mathrm{P}_{\text {trend }}$ & & & 0.015 & & $3.9 \times 10^{-6}$ & & 0.76 & \\
\hline Continuous $^{\dagger}$ & 441 & 185 & $1.49(1.09$ to 2.04$)$ & 58 & $4.51(2.59$ to 7.84$)$ & 127 & $1.01(0.72$ to 1.43$)$ & $<0.001$ \\
\hline \multicolumn{9}{|l|}{ Ang-2 } \\
\hline$<1714.0$ & 111 & 31 & 1.00 (ref) & 7 & 1.00 (ref) & 24 & 1.00 (ref) & \\
\hline $1714.0-2494.0$ & 110 & 50 & $2.11(1.12$ to 3.96$)$ & 10 & $1.79(0.60$ to 5.40$)$ & 40 & $2.25(1.14$ to 4.45$)$ & \\
\hline $2494.1-3545.7$ & 110 & 43 & $1.36(0.71$ to 2.59$)$ & 15 & $2.05(0.74$ to 5.72$)$ & 28 & $1.15(0.56$ to 2.36$)$ & \\
\hline$>3545.7$ & 110 & 61 & $2.14(1.15$ to 3.98$)$ & 26 & $4.25(1.60$ to 11.3$)$ & 35 & $1.64(0.82$ to 3.27$)$ & \\
\hline$P_{\text {trend }}$ & & & 0.07 & & 0.0017 & & 0.52 & \\
\hline Continuous $^{\dagger}$ & 441 & 185 & $1.27(0.98$ to 1.64$)$ & 58 & $1.98(1.33$ to 2.95$)$ & 127 & $1.06(0.79$ to 1.41$)$ & 0.0036 \\
\hline \multicolumn{9}{|l|}{ CXCL12 } \\
\hline$<1255.5$ & 111 & 46 & 1.00 (ref) & 9 & 1.00 (ref) & 37 & 1.00 (ref) & \\
\hline $1255.5-1612.2$ & 110 & 44 & $0.73(0.40$ to 1.35$)$ & 15 & $1.41(0.53$ to 3.72$)$ & 29 & $0.59(0.30$ to 1.15$)$ & \\
\hline $1612.3-1924.6$ & 110 & 38 & $0.68(0.37$ to 1.26$)$ & 16 & $1.91(0.72$ to 5.05$)$ & 22 & $0.46(0.23$ to 0.92$)$ & \\
\hline$>1924.6$ & 110 & 57 & $0.94(0.52$ to 1.71$)$ & 18 & $1.75(0.67$ to 4.59$)$ & 39 & $0.79(0.41$ to 1.52$)$ & \\
\hline$P_{\text {trend }}$ & & & 0.78 & & 0.27 & & 0.38 & \\
\hline Continuous $^{\dagger}$ & 441 & 185 & $1.02(0.73$ to 1.42$)$ & 58 & $1.37(0.82$ to 2.29$)$ & 127 & $0.93(0.65$ to 1.32$)$ & 0.16 \\
\hline \multicolumn{9}{|l|}{ CCL8 } \\
\hline$<27.3$ & 111 & 56 & 1.00 (ref) & 11 & 1.00 (ref) & 45 & 1.00 (ref) & \\
\hline $27.3-36.6$ & 110 & 48 & $0.91(0.51$ to 1.61$)$ & 16 & $1.4(0.57$ to 3.45 & 32 & $0.77(0.41$ to 1.45$)$ & \\
\hline $36.7-50.3$ & 110 & 43 & $0.91(0.50$ to 1.66$)$ & 15 & $1.63(0.64$ to 4.15$)$ & 28 & $0.75(0.38$ to 1.45$)$ & \\
\hline$>50.3$ & 110 & 38 & $1.03(0.55$ to 1.95$)$ & 16 & $2.02(0.78$ to 5.22$)$ & 22 & $0.79(0.39$ to 1.60$)$ & \\
\hline$P_{\text {trend }}$ & & & 0.89 & & 0.15 & & 0.53 & \\
\hline Continuous $^{\dagger}$ & 441 & 185 & $0.90(0.66$ to 1.23$)$ & 58 & $1.22(0.74$ to 2.01$)$ & 127 & $0.81(0.58$ to 1.15$)$ & 0.13 \\
\hline \multicolumn{9}{|l|}{ BMP-9 } \\
\hline$<94.4$ & 111 & 27 & 1.00 (ref) & 7 & 1.00 (ref) & 20 & 1.00 (ref) & \\
\hline $94.4-144.1$ & 110 & 35 & $1.19(0.60$ to 2.35$)$ & 8 & $0.84(0.27$ to 2.62$)$ & 27 & $1.41(0.66$ to 2.99$)$ & \\
\hline $144.2-213.7$ & 110 & 49 & $1.27(0.67$ to 2.40$)$ & 14 & $1.15(0.41$ to 3.25$)$ & 35 & $1.36(0.67$ to 2.77$)$ & \\
\hline$>213.7$ & 110 & 74 & $1.61(0.87$ to 3.00$)$ & 29 & $2.14(0.83$ to 5.57$)$ & 45 & $1.41(0.70$ to 2.84$)$ & \\
\hline$P_{\text {trend }}$ & & & 0.12 & & 0.025 & & 0.46 & \\
\hline Continuous $^{\dagger}$ & 441 & 185 & $1.18(1.00$ to 1.40$)$ & 58 & $1.26(0.95$ to 1.68$)$ & 127 & $1.16(0.97$ to 1.40$)$ & 0.59 \\
\hline
\end{tabular}

* Adjusted for age, sex, race, study year and calendar year of phlebotomy, and study center, as well as a score variable based on established risk factors for progression (M-protein concentration, serum free light-chain ratio, and Ig type) with categories defined as LC MGUS, non-IgM MGUS with no risk factors, and non-IgM MGUS with abnormal sFLC ratio and/or elevated $\mathrm{M}$-protein concentration.

$\dagger$ Estimated risk corresponding to an approximate doubling in marker levels (1-unit increase in $\log _{2}$-transformed levels). 
Table 4. Estimated absolute risk and measures of risk prediction for the angiogenesis marker score and progression from MGUS to multiple myeloma, stratified by characteristics of MGUS

\begin{tabular}{|c|c|c|c|}
\hline Measure & Light-chain MGUS & $\begin{array}{l}\text { Low risk non-IgM } \\
\text { MGUS }\end{array}$ & $\begin{array}{l}\text { Intermediate/high risk } \\
\text { non-IgM MGUS }\end{array}$ \\
\hline No. progressed/overall & $28 / 205$ & $47 / 193$ & $110 / 228$ \\
\hline \multicolumn{4}{|l|}{$\begin{array}{l}\text { Estimated absolute risk of } \\
\text { progression }(95 \% \mathrm{CI})\end{array}$} \\
\hline Low angiogenesis score & $0.2 \%$ (0 to $0.7 \%)$ & $0.6 \%(0.2$ to $1.5 \%)$ & $1.4 \%(0.7$ to $2.9 \%)$ \\
\hline High angiogenesis score ${ }^{*}$ & $2.6 \%(1.2$ to $5.2 \%)$ & $2.3 \%(1.0$ to $5.2 \%)$ & $8.3 \%(5.2$ to $13.0 \%)$ \\
\hline P-value & 0.012 & 0.10 & 0.0006 \\
\hline $\mathrm{AUC}^{\dagger}$ & 0.78 & 0.65 & 0.71 \\
\hline MRS/year & $0.19 \%$ & $0.11 \%$ & $0.61 \%$ \\
\hline NNtest ${ }^{\natural}$ & 1,059 & 1,745 & 330 \\
\hline Sensitivity ${ }^{\S}$ & $82.5 \%$ & $51.5 \%$ & $74.3 \%$ \\
\hline Specificityll & $72.8 \%$ & $77.8 \%$ & $67.0 \%$ \\
\hline \multicolumn{4}{|l|}{$\begin{array}{l}\text { 10-year follow-up: } \\
\text { Estimated absolute risk of } \\
\text { progression }(95 \% \mathrm{CI})\end{array}$} \\
\hline Low angiogenesis score ${ }^{*}$ & $1.0 \%(0.5$ to $1.9 \%)$ & $2.2 \%(1.3$ to $3.6 \%)$ & $6.4 \%(4.3$ to $9.4 \%)$ \\
\hline High angiogenesis score* & $4.2 \%(2.3$ to $7.6 \%)$ & $5.3 \%(2.8$ to $9.9 \%)$ & $15.3 \%$ (10.3 to $22.2 \%)$ \\
\hline P-value & 0.016 & 0.076 & 0.007 \\
\hline \multicolumn{4}{|l|}{$\begin{array}{l}\text { Predictiveness in years } 5- \\
10^{* *}:\end{array}$} \\
\hline $\mathrm{AUC}^{\dagger}$ & 0.58 & 0.57 & 0.54 \\
\hline MRS/year & $0.07 \%$ & $0.11 \%$ & $0.18 \%$ \\
\hline NNtest ${ }^{\natural}$ & 2,902 & 1,904 & 1,096 \\
\hline Sensitivity ${ }^{\S}$ & $43.1 \%$ & $36.0 \%$ & $41.3 \%$ \\
\hline Specificityll & $72.7 \%$ & $72.7 \%$ & $66.8 \%$ \\
\hline \multicolumn{4}{|l|}{$\begin{array}{l}\text { Estimated absolute risk of } \\
\text { progression }(95 \% \mathrm{CI})\end{array}$} \\
\hline Low angiogenesis score ${ }^{*}$ & $1.0 \%(0.5$ to $1.9 \%)$ & $3.5 \%(2.2$ to $5.4 \%)$ & $8.9 \%(6.2$ to $12.7 \%)$ \\
\hline High angiogenesis score & $4.2 \%(2.3$ to $7.6 \%)$ & $8.2 \%(4.7$ to $14.3 \%)$ & $18.3 \%$ (12.5 to $26.3 \%)$ \\
\hline P-value & 0.016 & 0.056 & 0.015 \\
\hline $\mathrm{AUC}^{\dagger}$ & 0.67 & 0.59 & 0.59 \\
\hline MRS/year & $0.13 \%$ & $0.12 \%$ & $0.30 \%$ \\
\hline NNtest ${ }^{\natural}$ & 1,529 & 1,607 & 660 \\
\hline Sensitivity ${ }^{\S}$ & $61.1 \%$ & $40.4 \%$ & $50.6 \%$ \\
\hline Specificity\|l & $72.8 \%$ & $77.8 \%$ & $66.9 \%$ \\
\hline
\end{tabular}

${ }^{*}$ The following equation was used to calculate the angiogenesis score:

$0.23 * \log _{2}(\mathrm{EGF})+0.21 * \log _{2}(\mathrm{HGF})+0.14 * \log _{2}($ Ang- 2$)$. We defined a high angiogenesis score as score $>5.25$ based on the cut point for the top tertile of the overall distribution; those in the lower tertiles were defined as having a low angiogenesis score.

$\dagger$ AUC, area under the curve (Receiver Operating Characteristic), for a binary test is the average of sensitivity and specificity.(38)

$¥$ MRS, mean risk stratification, is the average change in yearly MGUS progression rate to multiple myeloma revealed by the angiogenesis score.(18) Although AUC measures classification accuracy and MRS measures risk-stratification, AUC is related to MRS by the following equation: MRS $=4 *($ AUC -0.5$) * p^{*}(1-p)$, where $p$ is the yearly MGUS progression rate to multiple myeloma. Thus a group with a high AUC, but a low progression rate, might have less risk stratification than a low AUC but a high progression rate. For example, at 5-years of follow-up, light-chain MGUS has 
a superior AUC to intermediate/high-risk non-IgM MGUS (0.78 vs. 0.71), but less risk stratification (MRS of $0.19 \%$ vs. $0.61 \%$ progression per year to multiple myeloma). This is because the yearly progression rate to multiple myeloma is much lower for light-chain MGUS vs. intermediate/high-risk non-IgM MGUS $(0.17 \%$ vs. $0.74 \%)$.

" NNtest (which is computed as 2/MRS) is the number of individuals with MGUS who would need to be tested using the angiogensis panel to identify one additional case of progression to multiple myeloma per year of follow-up beyond those that would be identified by random selection. For example, we estimate that during the first five years of followup, 330 individuals with intermediate/high-risk MGUS would need to be tested with the angiogenesis panel to identify one additional case of progression to multiple myeloma per year. Although the AUCs for the angiogenesis panel are lower for intermediate/high-risk MGUS than for light-chain MGUS, this panel is more efficient at finding those who will progress to multiple myeloma among the intermediate/high-risk MGUS. This is attributable to the higher rate of progression in this group, which is taken into account by NNtest (and MRS) but not AUC.

$\S$ The fraction of multiple myeloma that occurred in those in the highest tertile of angiogenesis score

$\|$ The complement of the fraction of multiple myeloma that occurred in those in the bottom two tertiles of angiogenesis score

${ }^{* *}$ Reported values are restricted to the follow-up time period of 5-10 years after the blood draw at which MGUS and the immunologic markers were assessed. 
Figure 1. Summary of P-values from tests of association with MM risk for all markers

modeled as continuous variables. The top six markers were statistically significantly associated with MM using a false discovery rate of $10 \%$, and the top four markers met a Bonferroni-corrected $P<0.0011$. 
Figure 2. Estimated cumulative incidence of progression from MGUS to MM stratified by characteristics of MGUS and high vs. low levels of a composite angiogenesis marker score (based on levels of EGF, HGF, and Ang-2). MGUS groups were defined as follows: (A) LC MGUS ( $\left.\mathrm{N}_{\text {progressed }} / \mathrm{N}_{\text {overall }}=28 / 205\right)$, (B) non-IgM MGUS with no risk factors for progression $\left(\mathrm{N}_{\text {progressed }} / \mathrm{N}_{\text {overall }}=47 / 193\right)$, and (C) non-IgM MGUS with an elevated M-protein concentration $(\geq 15 \mathrm{~g} / \mathrm{L})$ and/or abnormal sFLC ratio $(<0.26$ or $>1.65)$

$\left(\mathrm{N}_{\text {progressed }} / \mathrm{N}_{\text {overall }}=110 / 228\right)$. The composite angiogenesis score was computed as follows: $0.23 * \log _{2}(\mathrm{EGF})+0.21 *$

$\log _{2}(\mathrm{HGF})+0.14 * \log _{2}($ Ang-2). A high angiogenesis marker score was defined as score $>5.25$ based on the cut point for the top tertile of the overall distribution; those in the lower tertiles were defined as having a low angiogenesis score. 


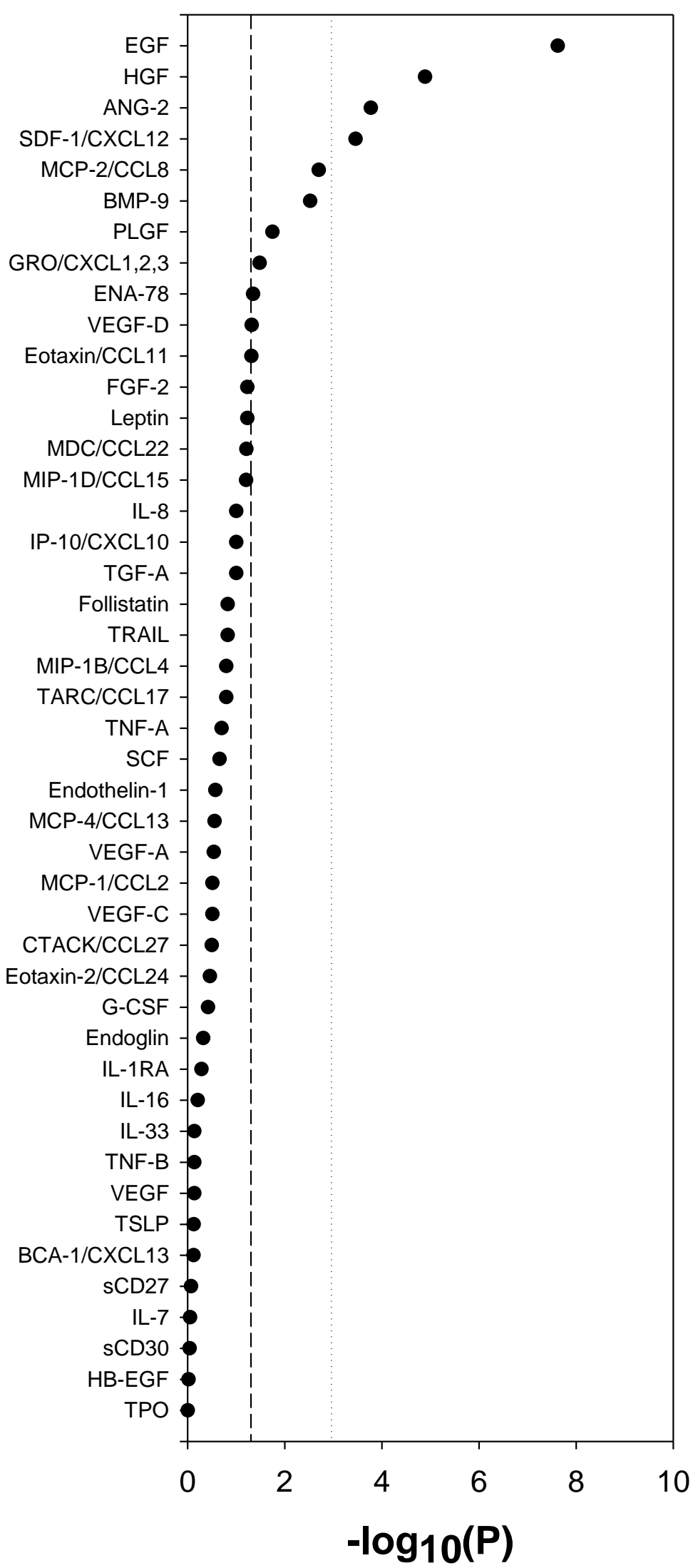




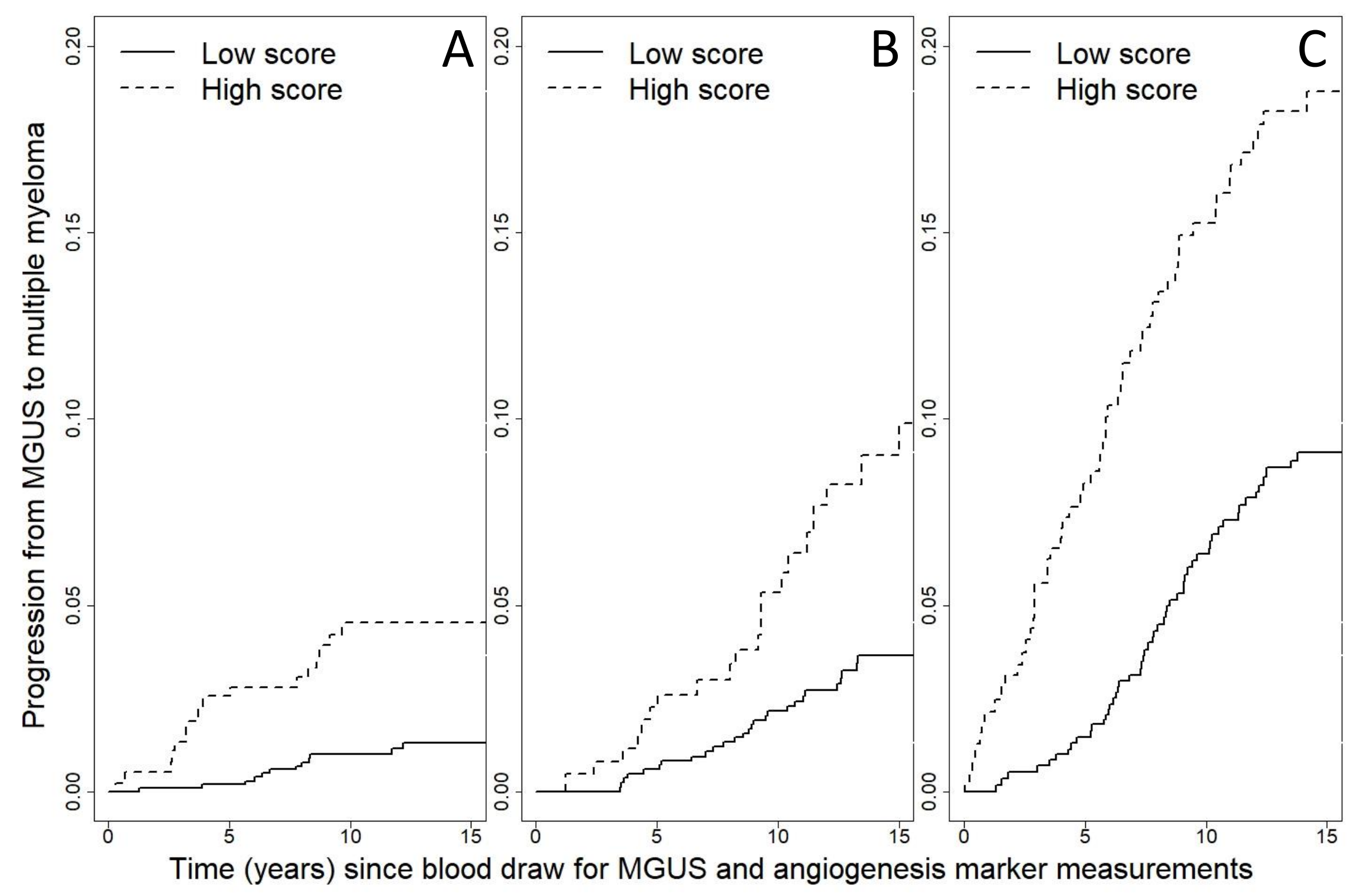

